\title{
EIGENVALUES EVALUATION OF GENERALLY DAMPED ELASTIC DISC BRAKE MODEL LOADED WITH NON-CONSERVATIVE FRICTION FORCES
}

\author{
Juraj ÚradníčEK ${ }^{a *}$, Miloš Musil $^{a}$, Michal BaChraty $^{b}$ \\ ${ }^{a}$ Slovak University of Technology, Faculty of Mechanical Engineering, Institute of Applied Mechanics and \\ Mechatronics, Námestie Slobody 17, 82131 Bratislava, Slovak republic. \\ ${ }^{b}$ Slovak University of Technology, Faculty of Mechanical Engineering, Institute of Manufacturing Systems, \\ Environmental Technology and Quality Management, Námestie Slobody 17, 82131 Bratislava, Slovak republic. \\ * corresponding author: juraj.uradnicek@stuba.sk
}

\begin{abstract}
This paper deals with the evaluation of eigenvalues of a linear damped elastic twodegrees-of-freedom system under a non-conservative loading. As a physical interpretation of a proposed mathematical model, a simplified disk brake model is considered. A spectral analysis is performed to predict an eigenvalues bifurcation, known as the Krein collision, leading to double eigenvalues, one of them having a positive real part causing a vibration instability of the mechanical systems. This defective behaviour of eigenvalues is studied with respect to a magnitude of non-conservative Coulomb friction force, through the variation of the friction coefficient. The influence of a proportional versus general damping on the system stability is further analysed. The generalized non-symmetric eigenvalue problem calculation is employed for spectral analyses, while a modal decomposition is performed to obtain a time-domain response of the system. The analyses are compared with an experiment.
\end{abstract}

KEYWORDS: Eigenvalues bifurcation, Krein collision, non-conservative force, modal decomposition, brake squeal.

\section{INTRODUCTION}

Brake systems represent one of the most important safety and performance components of vehicles, such as cars, trains, airplanes or industrial machines. Reliability, braking power and a fluent operation are important properties of brake systems. However, during the braking, the components of a disc brake tend to vibrate and a noise and harshness such as a brake squeal can occur. It is generally accepted that the brake squeal is determined by a friction-induced vibration via a rotating disc [1].

In 1952, Ziegler [2], studying a double mathematical pendulum exposed to the non-conservative force, found that the limits of the system stability were reduced by an application of minor damping to the system. Damping reduces the stability of the system, which contradicts the classical theory of structural stability, in which the damping plays a purely stabilizing role. Thus, an unstable behaviour of mechanical systems can also be caused by a dissipation induced instability, which was, in the case of brake system, pointed out in the work of Hoffman and Gaul in 2003 [3. In a braking system, the non-conservative friction force plays a major role. For this reason, the disc brake system is considered as the system with a potential occurrence of the damping destabilization paradox.

Several studies focus on analyses of the behaviour of such systems using simple analytical minimal models [4, 5], complex numerical Finite Element (FE) mod- els 11 and experiments on real physical systems [6]. Analytical and FE studies are mainly based on the complex Eigenvalue Analysis [7] to predict the mode coupling. These analyses subsequently allow an optimization of brake systems to avoid a potential brake squeal occurrence.

In the process of disc brake development, the complex eigenvalue analysis of finite element model is usually used to perform the modal optimization of the system to reduce any potential system instability [8. In this process, no damping or simple proportional damping is usually considered. According to the above-mentioned destabilization paradox, a damping can possibly cause a reduction of stability limits. Significance of this effect should be closely examined for disc brake applications.

In this paper, the damping in the system and its influence on the system stability is studied on a simple two-degree-of-freedom (DOF) analytical model of a disc brake. Observations are experimentally verified by measurements on an experimental pad-on-disc system.

\section{PhysicAl PROBLEM DESCRIPTION}

In the following study, a simple two-degree-of-freedom elastic system is considered (Figure 1). The system consists of spring/damping elements and a mass moving in the horizontal and vertical direction. The spring and damping elements in a skew direction couple both degrees of freedom so that the mode local bifurcation 


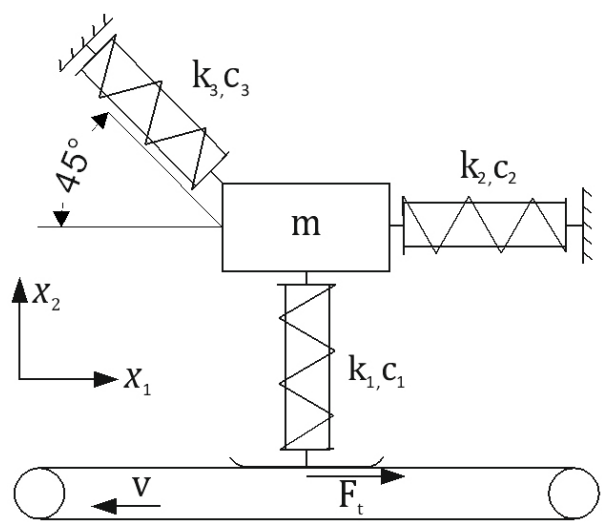

Figure 1. Two-degrees-of-freedom mechanical model including non-conservative friction force in the system equilibrium position.

of complex conjugate eigenvalues (mode coupling) can occur [9]. The mass element represents the mass of the friction material (a part of the braking pad which is in contact with the disc), while spring/damping elements represent the stiffness and damping of the friction material in particular directions. The moving belt acts as a generator of the non-conservative friction force whose magnitude depends linearly on state variables and a coefficient of friction, considering Coulomb friction law. To avoid the nonlinear behaviour of the friction force related to changing its sign, the following assumption is taken into account. The solution is evaluated around the equilibrium point. This case corresponds to the situation when the prestress effect is applied on the mass in a negative $x_{2}$ direction and after the solution is obtained around this system's equilibrium point. Under this assumption, the overall friction force can exhibit only positive values. In this paper, the evaluation of the eigenvalues with respect to the friction coefficient is studied to investigate the limits of stability of the generally damped simple minimal brake model.

\section{Mathematical PROBlem DESCRIPTION}

The mechanical system in Figure 1 is described by a set of second order differential equations in matrix form

$$
\begin{gathered}
\mathbf{M} \ddot{\mathbf{x}}+\mathbf{C} \dot{\mathbf{x}}+\mathbf{K} \mathbf{x}=\mathbf{F} \\
\mathbf{M}=\left[\begin{array}{cc}
m & 0 \\
0 & m
\end{array}\right], \mathbf{C}=\left[\begin{array}{cc}
c_{2}+\frac{1}{2} c_{3} & \frac{1}{2} c_{3} \\
\frac{1}{2} c_{3} & c_{1}+\frac{1}{2} c_{3}
\end{array}\right], \\
\mathbf{K}=\left[\begin{array}{cc}
k_{2}+\frac{1}{2} k_{3} & \frac{1}{2} k_{3} \\
\frac{1}{2} k_{3} & k_{1}+\frac{1}{2} k_{3}
\end{array}\right], \mathbf{F}=\left[\begin{array}{c}
F_{t} \\
0
\end{array}\right], \mathbf{x}=\left[\begin{array}{l}
x_{1} \\
x_{2}
\end{array}\right],
\end{gathered}
$$

where coordinates $x_{1}, x_{2}$ represent the displacement of the mass around the equilibrium position, $\mathbf{M}, \mathbf{B}, \mathbf{K}$ are coefficients' matrices and $F_{t}$ represents the dynamical part of the overall friction force $F_{t c}=F_{o}+F_{t}$.
Constant friction force $F_{o}=\mu F_{p}$ results from the pre-stress normal force $F_{p}$ and inequality $F_{p}>\left|F_{t}\right| / \mu$ has to be fulfilled so that the slider cannot detach the moving belt. If the Coulomb friction model is considered and the slider in the model cannot detach the moving belt, the friction force $F_{t}$ linearly depends on the normal force acting between the slider and the moving belt:

$$
F_{t}=\mu\left(k_{1} x_{2}+c_{1} \dot{x}_{2}\right)
$$

where the $\mu$ represents the Coulomb friction coefficient. Substituting (2) into (1), the system is reorganized to the form

$$
\begin{gathered}
\left\{\begin{array}{l}
\mathbf{M} \ddot{\mathbf{x}}+\mathbf{C}^{\prime}(\mu) \dot{\mathbf{x}}+\mathbf{K}^{\prime}(\mu) \mathbf{x}=\mathbf{0} \\
\mathbf{M} \dot{\mathbf{x}}-\mathbf{M} \dot{\mathbf{x}}=\mathbf{0}
\end{array}\right. \\
\mathbf{K}^{\prime}=\left[\begin{array}{cc}
k_{2}+\frac{1}{2} k_{3} & \frac{1}{2} k_{3}-\mu k_{1} \\
\frac{1}{2} k_{3} & k_{1}+\frac{1}{2} k_{3}
\end{array}\right] \\
\mathbf{C}^{\prime}=\left[\begin{array}{cc}
c_{2}+\frac{1}{2} c_{3} & \frac{1}{2} c_{3}-\mu c_{1} \\
\frac{1}{2} c_{3} & c_{1}+\frac{1}{2} c_{3}
\end{array}\right]
\end{gathered}
$$

Adding the identical equation in (3), the system is transformed into the state-space representation with state variables vector $\mathbf{y}$,

$$
\begin{gathered}
\mathbf{A}(\mu) \dot{\mathbf{y}}+\mathbf{B}(\mu) \mathbf{y}=\mathbf{0} \\
\mathbf{A}=\left[\begin{array}{cc}
\mathbf{C}^{\prime} & \mathbf{M} \\
\mathbf{M} & \mathbf{0}
\end{array}\right], \mathbf{B}=\left[\begin{array}{cc}
\mathbf{K}^{\prime} & \mathbf{0} \\
\mathbf{0} & -\mathbf{M}
\end{array}\right], \mathbf{y}=\left[\begin{array}{c}
\mathbf{x} \\
\dot{\mathbf{x}}
\end{array}\right] .
\end{gathered}
$$

It can be seen that matrices $\mathbf{A}$ and $\mathbf{B}$ are nonsymmetric and smoothly depend on the scalar parameter $\mu$.

\section{System eigenvalues evaluation}

The equation (4) can be rewritten in the form of a generalized non-symmetric eigenvalue problem,

$$
\left(\mathbf{B}+\lambda_{i} \mathbf{A}\right) \mathbf{v}_{r i}=\mathbf{0}
$$

where $\lambda_{i}$ is the $i-t h$ generalized eigenvalue and $v_{r i}$ is the corresponding $i-t h$ right complex eigenvector. An imaginary part of the eigenvalue is the damped natural frequency $\omega_{d}=\omega_{0} \sqrt{1-\zeta^{2}}$, where $\omega_{0}$ represents an undamped natural frequency and the real part is $\zeta \omega_{0}$. A damping ratio $\zeta$ is calculated from a geometrical relation $\cos \theta=\zeta$, where $\theta$ is an angle to a pole from a negative real axis.

In the undamped case, the local bifurcation of the complex conjugate eigenvalues occurs in the point $\mu=0.5$ (Figure 2 and the damped natural frequencies are being coupled (Figure 3). At this point, the system loses its stability. In the case of the proportional damping, the system loses its stability slightly over the point $\mu=0.5$ and damped natural frequencies cross each other but don't couple. In the case of the general non-proportional damping, the bifurcation point is unclear and the system loses its stability below $\mu=0.5$. 


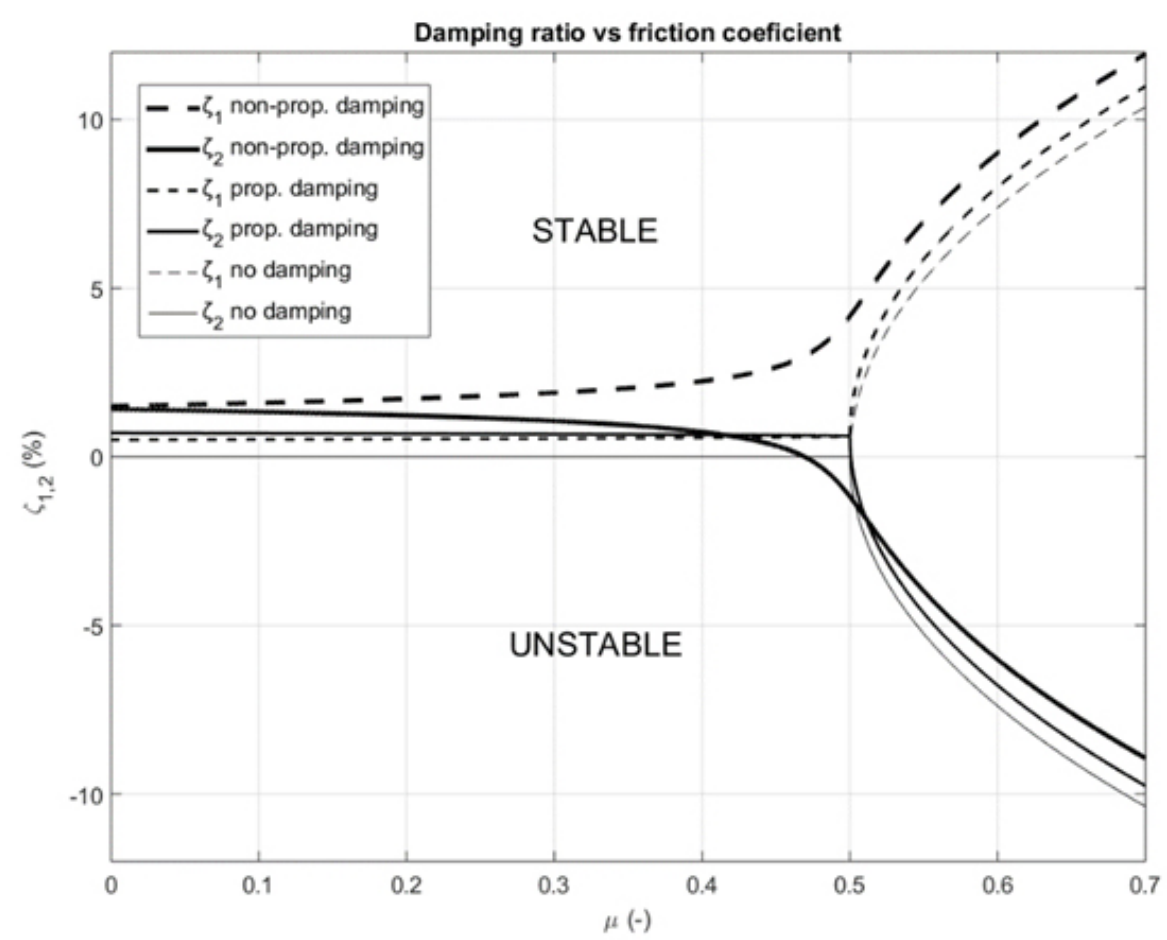

FiguRE 2. 1. and 2. modes damping ratios' evolution with the friction coefficient, considering the proportionally damped, non-proportionally damped and undamped system.

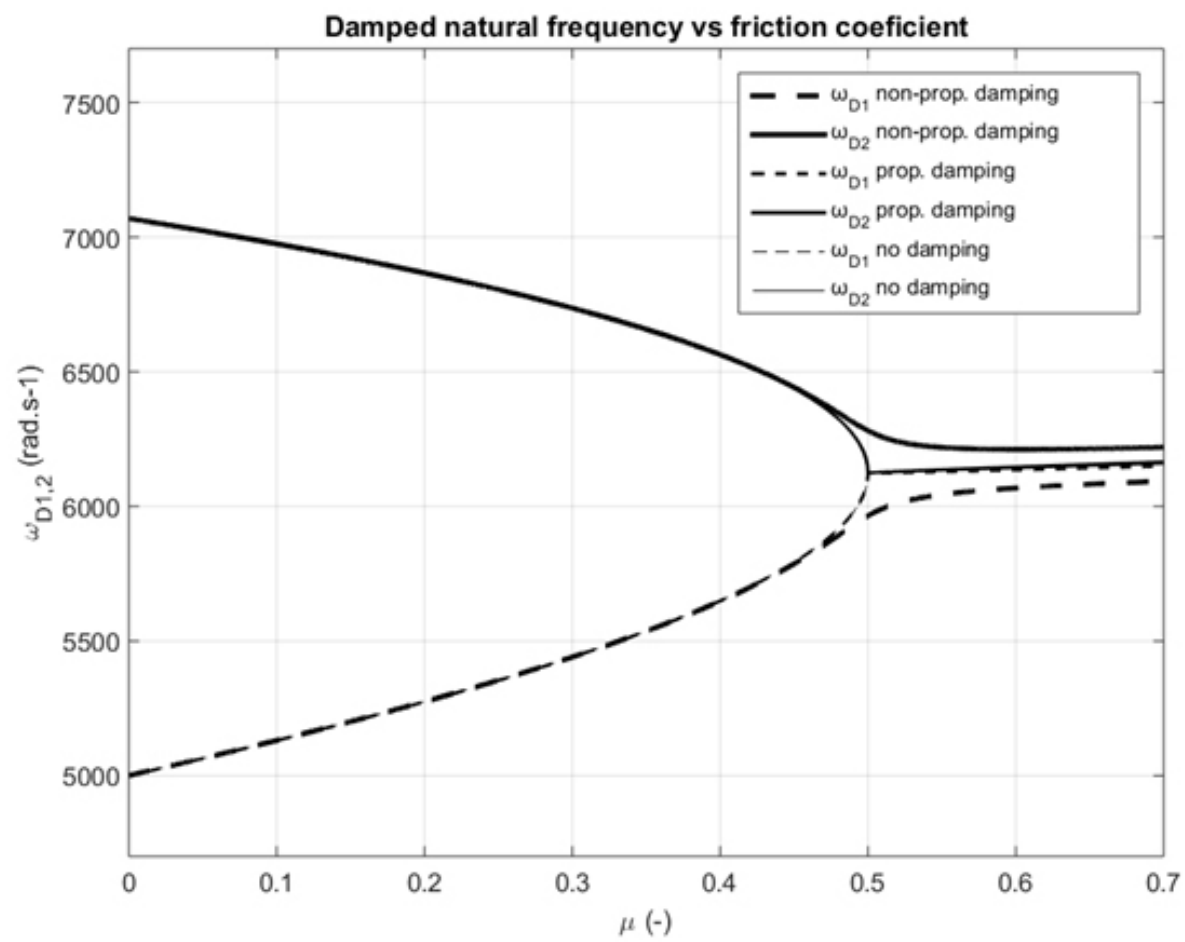

FiguRE 3. Damped natural frequencies' evolution of the 1. and 2. modes with the friction coefficient considering proportionally damped, non-proportionally damped and non-damped system. 


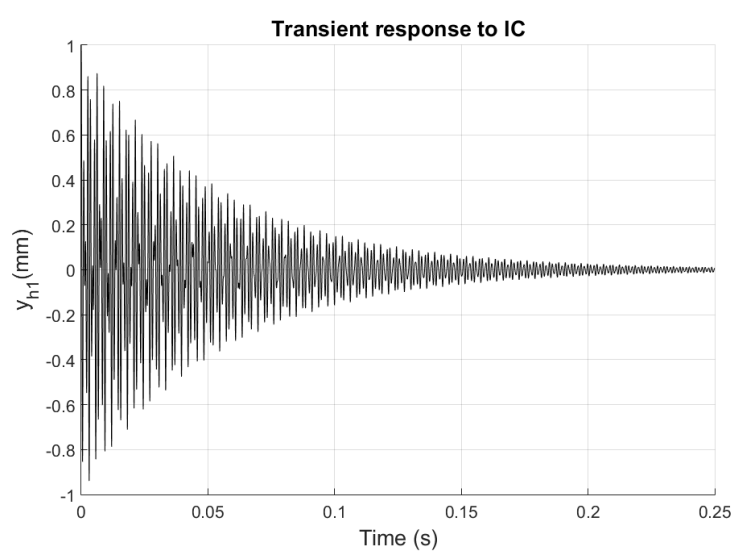

(A).

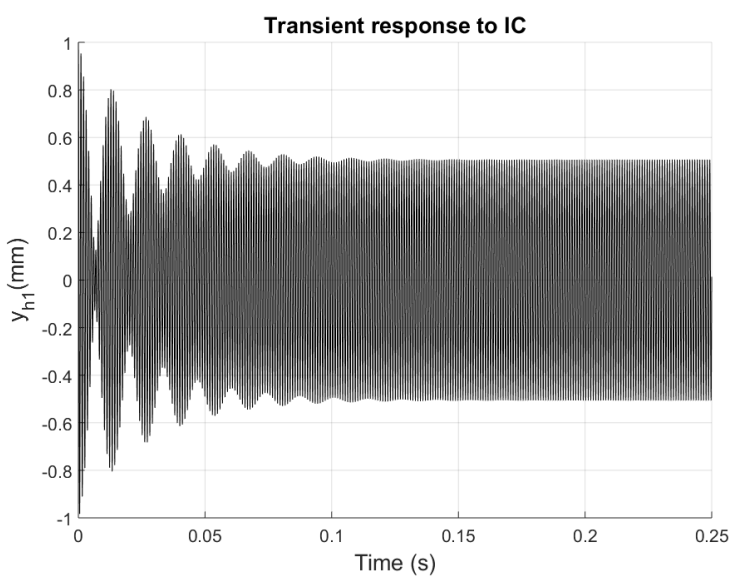

(C).

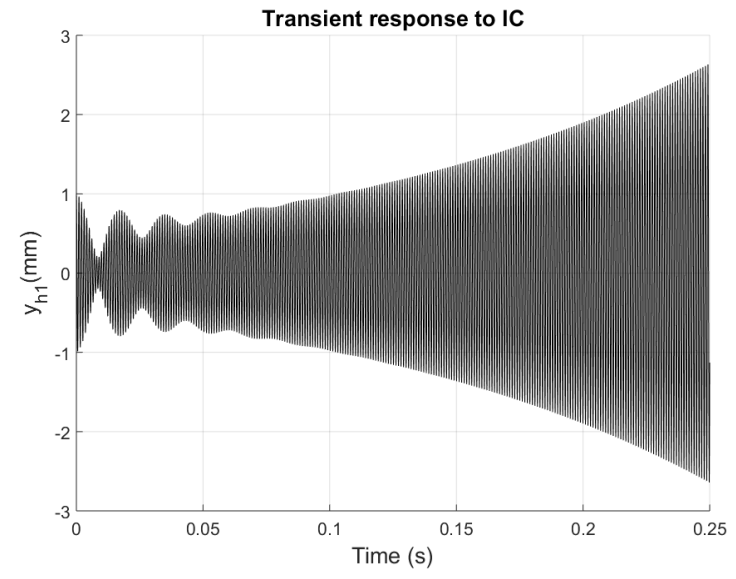

(E).

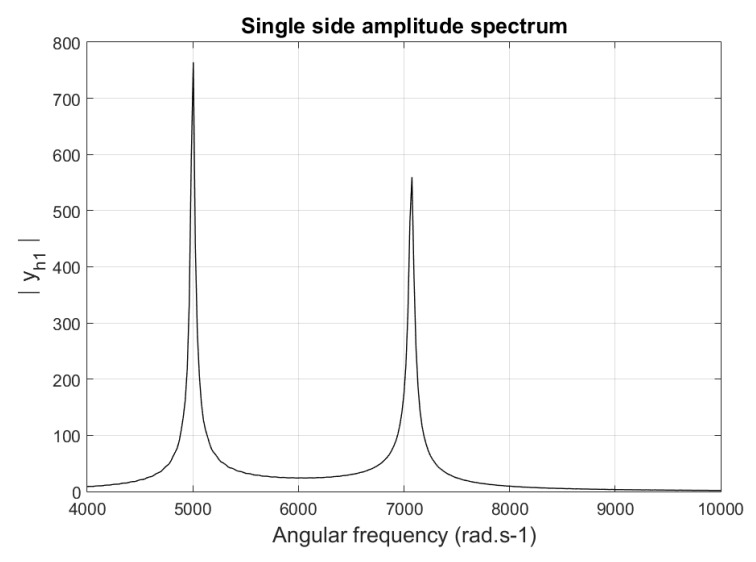

(B).

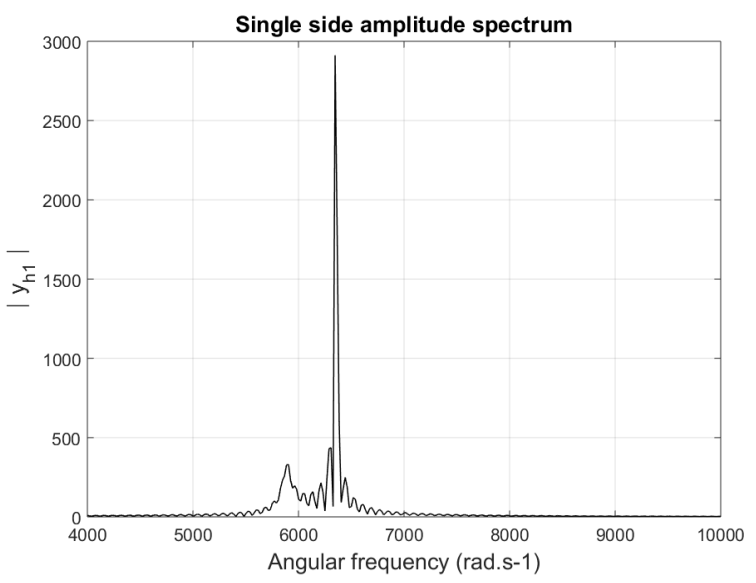

(D).

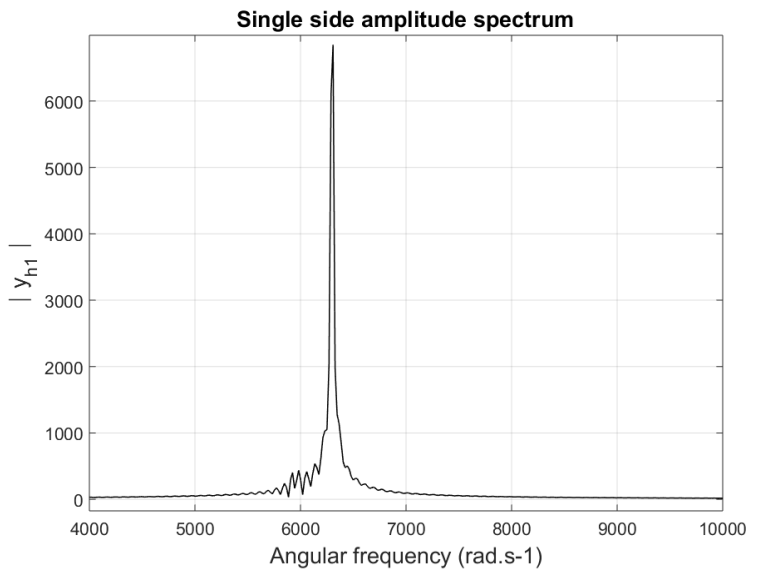

(F).

FigURE 4. The transient response of the non-proportionally damped system to the initial conditions $($ IC) $\mathbf{y}(0)=$ $[1,0,0,0]$ for a) $\mu=0\left(\lambda_{1}=-15.6 \pm 5000 i ; \lambda_{2}=-21.9 \pm 7071 i\right)$, b) $\mu=0.47\left(\lambda_{1}=-37.5 \pm 5886 i ; \lambda_{2}=0 \pm 6352 i\right)$, c) $\mu=0.48\left(\lambda_{1}=-44 \pm 5943 i ; \lambda_{2}=6.6 \pm 6300 i\right)$.

\section{ModAl DECOMPOSITION}

Homogeneous response of the linear state determined system can be expressed in terms of state-transition matrix $\Phi(t)$ as follows

$$
\mathbf{y}_{h}(t)=\Phi(t) \mathbf{y}(0)
$$

where the state-transition matrix $\Phi(t)$ may be expressed in terms of the eigenvalues/eigenvectors as

$$
\Phi(t)=\mathbf{V}_{r} e^{\lambda t} \mathbf{V}_{r}^{-1},
$$

defined by the modal matrix, consisting of right eigenvectors in columns, where $n$ is a number of columns of the modal matrix

$$
\mathbf{V}_{r}=\left[\begin{array}{l|l|l|l}
\mathbf{v}_{r 1} & \mathbf{v}_{r 2} & \cdots & \mathbf{v}_{r n}
\end{array}\right]
$$


and the square matrix $e^{\lambda t}$ with terms $e^{\lambda_{i} t}$ on the leading diagonal. Define

$$
\mathbf{R}_{r}=\mathbf{V}_{\mathbf{r}}{ }^{-1}\left[\begin{array}{c}
\mathbf{r}_{r 1} \\
\hline \mathbf{r}_{r 2} \\
\cdots \\
\hline \mathbf{r}_{r n}
\end{array}\right]
$$

the homogeneous response of the system can be expressed as a weighted superposition of the system modes $e^{\lambda_{i} t} \mathbf{v}_{r i}$ where an initial condition $\mathbf{y}(0)$ affects the strength of an excitation of each mode

$$
\begin{aligned}
\mathbf{y}_{h}(t) & =\left(\mathbf{V}_{r} e^{\lambda t}\right)\left(\mathbf{R}_{r} \mathbf{y}(0)\right) \\
& =\sum_{i=1}^{n} e^{\lambda_{i} t} \mathbf{v}_{r i}\left(\mathbf{r}_{r i} \mathbf{y}(0)\right) \\
& =\sum_{i=1}^{n} c_{i} e^{\lambda_{i} t} \mathbf{v}_{r i}
\end{aligned}
$$

where the scalar coefficient $c_{i}=\mathbf{r}_{r i} \mathbf{y}(0)$.

The $y_{h 1}$ coordinate in Figure 4 represents the homogeneous response of the mass displacement $x_{1}$ in Figure 1. If the friction is absent in the system, both modes are attenuated over the time (Figure 4 a)) with the rate given by their damping ratios $\zeta_{i}$ derived from the corresponding eigenvalues $\lambda_{i}$. Damped eigenfrequencies $\omega_{D 1}=5000 \mathrm{rad} \cdot \mathrm{s}^{-1}$ and $\omega_{D 2}=7071 \mathrm{rad} \cdot \mathrm{s}^{-1}$ can be read from a Single Side Amplitude Spectrum (SSAS) and must correspond to given eigenvalues. If the friction coefficient reaches stability threshold, the negative real part of the corresponding eigenvalue changes its sign - the mode with a negative real part of $\lambda_{i}$ is attenuated over the time, while the mode with a positive real part becomes unstable and is increasing or maintaining (if real part is 0) its amplitude (Figure 4 b)). It can be seen that the mode with the lower frequency is attenuated while the second one with the higher frequency becomes unstable. Damped eigenfrequencies $\omega_{D 1,2}$ become closer to each other with increasing coefficient $\mu$. A last plot (Figure 4 c)) shows the response for the friction coefficient set over the stability threshold value. From eigenvalues $\lambda_{i}$, it can be seen that the stable mode is attenuated faster (compared to b) case) due to the lower real part value. Oppositely, the unstable mode is increasing its amplitude faster over the time due to the higher value of the positive real part. The SSAS shows that the unstable mode fully dominates the stable one over the analysed time interval.

\section{EXPERIMENTAL RESULTS AND DISCUSSION}

To investigate the existence of described effects in a real application, a simple experimental device has been set up. The device consists of a simplified padon-disc system where a friction material is pressed onto a rotating disc. Elastic properties of a pad are achieved through a thin plate attached between the
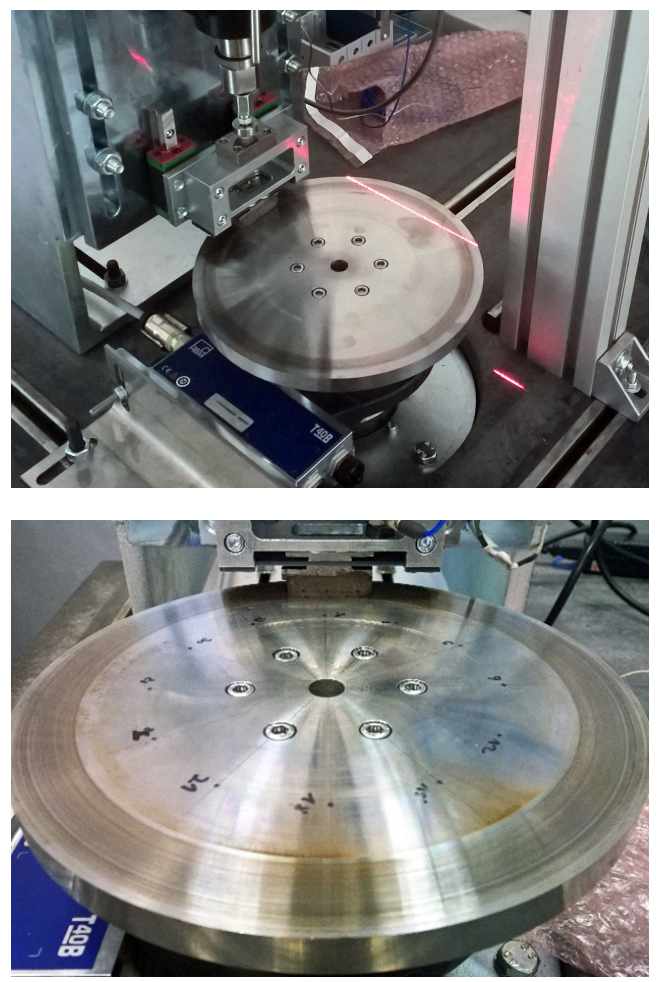

Figure 5. Experimental pad-on-disc set up.

friction material and a piston of a linear motor which generates a normal force.

Dynamical instability and self-excited vibrations of the system lead to a brake squeal. This effects can be measured both using accelerometer sensors attached to the flexible structure and a microphone, which directly measures the sound pressure as a side effect of an extensive vibration of the system. The time evaluation of the sound pressure (Figure 6) carries out the information about an intensity of a the vibration and also a spectral content of the vibration. The spectral content is observed through a single side amplitude spectrum by the Fourier transformation of the sound pressure time signal (Figure 7).

In a real physical environment, more physical aspects are involved in the process of dynamic destabilization. It can be demonstrated that nonlinear effects in friction contact, such as stick slip [10] or contact surfaces detaching [11, lead into limit cycles of vibrations. These effects bring perturbations to the system. If the system is perturbed, both stable and unstable eigenmodes should be excited over the time. This behaviour can be seen from the experimental observed data from the real physical system. The experimental data have been studied over the time interval of $0.06 \mathrm{~s}$. The variation of the vibration amplitude can be seen in Figure 6 The variation of the amplitude shows that one or more modes consisting in the response are changing their vibration propensity. This can be due to the combination of nonlinear effects, such as arising the amplitudes of unstable modes into limit cycles along with the attenuation of the repeatedly excited stable modes. Strong nonlinear effects on the signal 


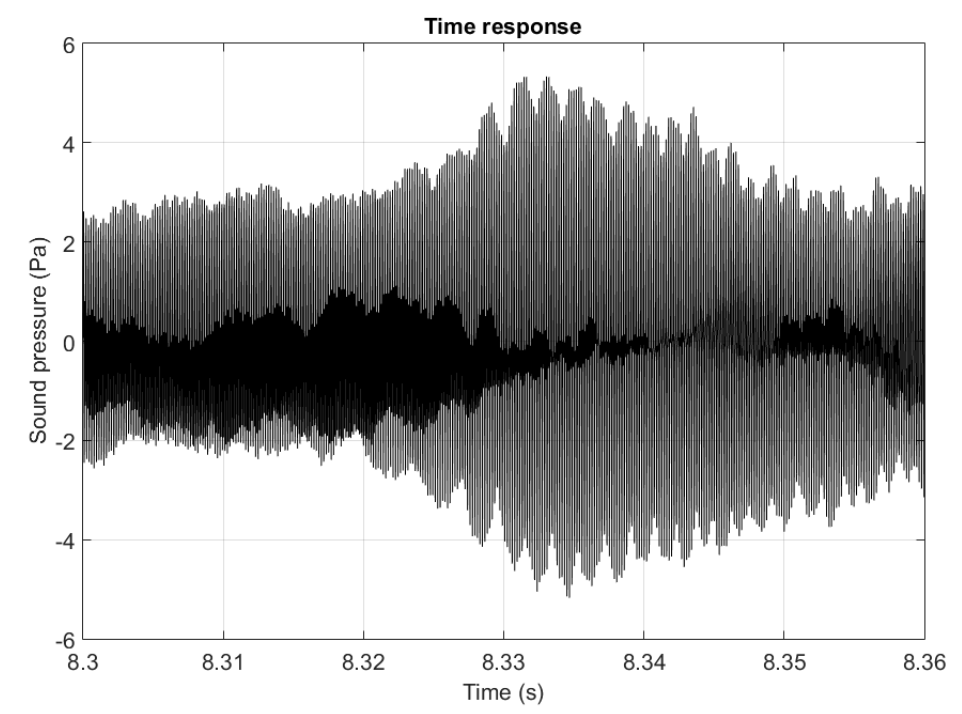

FiguRE 6 . Sound pressure evaluation over the time interval of $0.06 \mathrm{~s}$.

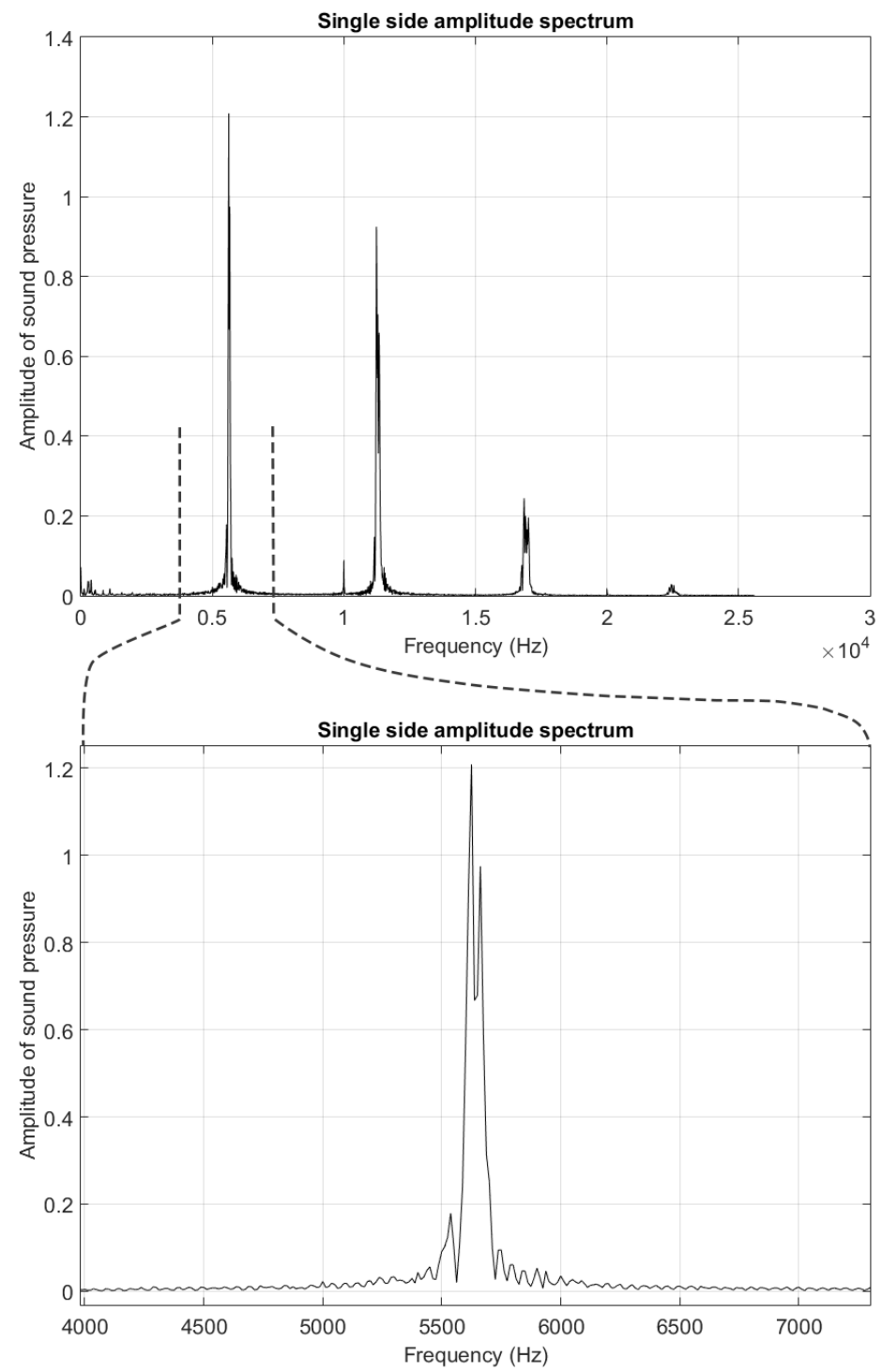

Figure 7. Single side amplitude spectrum of the sound pressure over the studied time interval of the length $0.06 \mathrm{~s}$. 
can be concluded from the poly-harmonic response of the signal in Figure 7 consisting of the fundamental frequency of $5700 \mathrm{~Hz}$ and its higher harmonics. A detailed observation of the signal spectrum around $5700 \mathrm{~Hz}$ shows more harmonics with relatively close frequencies contained in the signal. This can be due to the upper mentioned bifurcation effects. Coexistence of more than one eigenmode in the unstable state points out the effect of non-proportional damping in the system, which has been described in the previous chapter.

\section{Conclusions}

The destabilization paradox due to the nonconservative friction force in the system with nonproportional damping can play a significant role, since the evolution of system eigenvalues is different when compared to the proportionally damped or undamped systems. The stability threshold value of the friction decreases with the non-proportional damping. Damped eigenfrequencies don't couple, more precisely, do not cross each other in the bifurcation point in comparison to the undamped system and proportionally damped systems. This behaviour is known, in various physical problems, as the destabilization paradox or the dissipation induced destabilization. Even the described model represents only the mode coupling mechanism of the linear system where an unstable mode cannot coexists with a stable one in the steady solution, the experimental observation proved the coexistence of more than one mode in the response of the unstable (squealing) system. This coexistence of double modes points out on the general (non-proportional) damping or a system non-linearity. The cause of double modes in experimental analyses should be closely examined by the measurement of damping properties of the system to determine how significantly non-proportional the damping is and how this non-proportionality can reduce the stability threshold value. The non-proportional damping is expected due to significantly different material properties of the friction material and the rest of the system [12. This discussion leads to the question how much important role a general damping plays in a brake squeal. The significance of these effects in brake systems will be further investigated in a next research.

\section{ACKNowledGements}

The research in this paper was supported by the grant agency VEGA 1/0227/19 of Ministry of Education, Science and Sport of the Slovak Republic and the author also appreciates the financial support provided by Slovak Research and Development Agency for the project APVV-0857-12.

\section{REFERENCES}

[1] R. A. Abu-Bakar, H. Ouyang. Recent studies of car disc brake squeal. In New Research on Acoustics, chap. 4. 2008 Nova Science Publishers, Inc., 2008.

[2] H. Ziegler. Die stabilitätskriterien der elastomechanik. Ing-Arch Springer Verlag 20:49-56, 1952.

[3] N. Hoffman, L. Gaul. Effects of damping on mode-coupling instability in friction induced oscillations. ZAMM Journal of applied mathematics and mechanics 83(8):524-534, 2003. DOI:10.1002/zamm.200310022.

[4] N. Hoffman, L. Fischer, et al. A minimal model for studying properties of the mode-coupling type instability in friction induced oscillations. Mechanics Research Communications 29(4):197-205, 2002. DOI:10.1016/S0093-6413(02)00254-9

[5] U. Wagner, D. Hochlenert, P. Hagedorn. Minimal models for disk brake squeal. Journal of Sound and Vibration 302(3):527-539, 2007. DOI:10.1016/j.jsv.2006.11.023.

[6] U. Wagner, T. Jearsiripongkul, et al. Brake squeal: Modelling and experiments. VDI-Bericht 1749:96-105, 2003.

[7] P. Liu, H. Zheng, et al. Analysis of disc brake squeal using the complex eigenvalue method. Applied Acoustics 68(6):603-615, 2007. DOI:10.1016/j.apacoust.2006.03.012

[8] J. Úradníček, M. Musil, P. Kraus. Predicting the self-excited vibrations in automotive brake systems. Noise and vibration in practice : peer-reviewed scientific proceedings 23(1):101-106, 2018.

[9] O. N. Kirillov. Nonconservative stability problems of modern physics. First printing. De Gruyter, Berlin, 2013. DOI:10.1515/9783110270433

[10] J. Úradníček, M. Musil, P. Kraus. Investigation of frictional stick-slick effect in disk brake nvh. Journal of Mechanical engineering 67(1):93-100, 2017. DOI:10.1515/scjme-2017-0010.

[11] J. Brunetti, F. Massi, W. D‘Ambrogio, et al. A new instability index for unstable mode selection in squeal prediction by complex eigenvalue analysis. Journal of Sound and Vibration 377(1):106-122, 2016. DOI:10.1016/j.jsv.2016.05.002

[12] P. Peciar, O. Macho, E. Eckert, et al. Modification of powder material by compaction processing. Acta Polytechnica 57 (2):116-124, 2017. ISSN 1805-2363, DOI:10.14311/AP.2017.57.0116. 